\title{
An Unexpected Presentation of Tuberculosis in the Surgical Field with a Preventable Disease Transmission
}

\author{
Hector M. Lopez ${ }^{1}$, Paloma M. Lugo ${ }^{1}$, Francisco J. Jacome ${ }^{2,3}$ \\ ${ }^{1}$ School of Medicine, University of Medicine and Health Sciences, Basseterre, Saint Kitts and Nevis \\ ${ }^{2}$ Department of Surgery, Doctors Hospital, Augusta, GA, USA \\ ${ }^{3}$ Department of Surgery, University Hospital Summerville, Augusta, GA, USA \\ Email: ${ }^{\star h m}$ lopez7@gmail.com
}

How to cite this paper: Lopez, H.M., Lugo, P.M. and Jacome, F.J. (2018) An Unexpected Presentation of Tuberculosis in the Surgical Field with a Preventable Disease Transmission. Surgical Science, 9, 407-411.

https://doi.org/10.4236/ss.2018.911047

Received: October 12, 2018

Accepted: November 23, 2018

Published: November 26, 2018

Copyright $\odot 2018$ by authors and Scientific Research Publishing Inc. This work is licensed under the Creative Commons Attribution International License (CC BY 4.0).

http://creativecommons.org/licenses/by/4.0/

\begin{abstract}
Tuberculosis (TB) incidence within Healthcare workers remains one of the lowest occupation specific rates in the United States. We report a case of a 33-year-old African American male presented with a right chest mass and without fever, chills or cough. Bacterial and Fungal cultures were sent during the initial visit and were negative throughout the entire management of his care. After several months of surgical interventions, the patient appeared positive for TB; however a staff member contracted the disease. The patient was managed successfully with the four-drug regimen of Isoniazid, Rifampin, Pyrazinamide and Ethambutol and recovered well from his surgeries.
\end{abstract}

\section{Keywords}

Tuberculosis, Transmission, Surgical Field, Prevention

\section{Introduction}

The number of reported cases of Tuberculosis (TB) has adopted a descending pattern after the temporary incidence escalation that took place in the late $80 \mathrm{~s}$ [1]. Although precautions are constantly taken to avoid the contraction of infectious diseases within Healthcare workers (HW), the reported percentage of TB cases in this population reached $3.9 \%$ in 2015 [1]. When comparing this rate to Correctional employees (CE), it was reported that only $0.1 \%$ of this population contracted TB (Table 1). Although most of the HWs that have higher risks of exposure (physicians, registered nurses, etc.) possess more medical knowledge than $\mathrm{CE}$, the rates still significantly higher regardless of aggressive preventative 
Table 1. Number of occupational tuberculosis cases by location and percentage by occupation in 2015.

\begin{tabular}{cccc}
\hline \multirow{2}{*}{ Location } & \multicolumn{3}{c}{ Case Distribution by Occupation } \\
\cline { 2 - 4 } & Total Reported Cases $(n)$ & Healthcare Workers (\%) & Correctional Employees (\%) \\
\hline United States & 9115 & 3.9 & 0.1 \\
Georgia & 306 & 3.4 & 0 \\
\hline
\end{tabular}

Comparison of nationwide occupational exposure rates with the state of Georgia, where this case occurred [1].

measures and compulsory screenings [2]. These findings can be attributed to three potential reasons: 1) the preventative measures are not being effective, 2) the HWs are not adequately complying with the protective guidelines or 3) both. However, these values might be due to pure casualty or infection contraction outside the work place (e.g. traveling). Thanks to the advents of molecular diagnostic tools and cough monitoring within healthcare settings, it is unusual to have patients admitted to hospitals with undiagnosed TB [3].

It is important to consider that amongst the subjects that take part of the HW, there are certain parties that encounter potential exposures on a daily basis, and within these subjects surgeons become one of the top affected sectors prone to healthcare associated hazards. Given the nature of their specialty, both attending physicians as well as surgical residents have greater risks of contracting not only air-borne infections, but also sharps injuries, latex allergies, blood-borne infections, anesthetic toxicity and so forth [4].

\section{Case Report}

We report a case of a 33-year-old African-American male that was referred to our practice due to a mass located at the right medial chest that had been progressively growing over the past three weeks. Ultrasound and Chest X-Rays were ordered by his Primary Care Physician, which showed a complex fluid collection of approximately $8 \times 4 \mathrm{~cm}$, a fractured underlying rib and chronic bibasilar scarring, respectively. Upon examination the patient denied fever, chills, cough and the mass, which was subsequently aspirated, had no overlying erythema but was tender to palpation. Bacterial and fungal cultures were sampled and resulted in no-growth throughout the whole management period. Laboratories were within normal limits.

The patient returned for a two-week follow-up and the aspirated mass re-accumulated fluid, requiring an Incision and Drainage in the operating room (OR). At the OR, the $10 \times 10 \times 10 \mathrm{~cm}$ cavity was assessed via digital exploration and a right $4^{\text {th }}$ rib fracture was confirmed. Negative-Pressure Wound Therapy (NPWT) was placed and set to $125 \mathrm{mmHg}$ of suction. NPWT sponges were changed every two days by one of our Certified Medical Assistants (CMA) over the course of three weeks.

Further investigation with Computed Tomography (CT) scan was indicated at 
this point. However, the uninsured status of this patient delayed CT imaging until a financial relief application was approved. After a month from the initial visit, a CT scan was done. As portrayed in Figure 1, the scan showed destructive changes of the anterior right $4^{\text {th }}$ rib, diffuse bilateral mediastinal lymphadenopathy, chronic interstitial changes of the Lung parenchyma and an abscess involving the pectoral muscles and extending to the right Lung margin. A Cardiothoracic surgeon (CTS) was consulted after the CT scan findings, for which he proceeded to operate for further debridement. Bone biopsies, smears and cultures were sampled, this time yielding a positive result for Acid Fast Bacilli. The patient was managed successfully with the four-drug regimen of Isoniazid, Rifampin, Pyrazinamide and Ethambutol and recovered well from the CTS intervention.

After notification of these results, a TB Skin Test was administered to the office staff for which unfortunately, the CMA that periodically changed the patient's dressings tested positive. Given such findings, the patient was re-interviewed more thoroughly and it was found that three weeks before the initial visit the patient was forcefully coughing to the point that he broke one of his ribs, leading to the whole cascade of events that initiated with the chest mass.

\section{Discussion}

As the incidence of TB in the United States decreases, it has become more common to assess what are the causes that might be leading to the cases that are being reported [1]. TB is commonly transmitted by air-borne mechanism via droplets that are released as the patient coughs or sneezes [3]. The effectiveness of this transmission is augmented when the patients are in close proximity or within small spaces like clinics, refugee camps, etc. Transmission also takes place in scenarios in which wound irrigation or debridement is required, in which surgical personnel might become susceptible [3].

Given that no trauma was reported in this case, it is thought that this patient

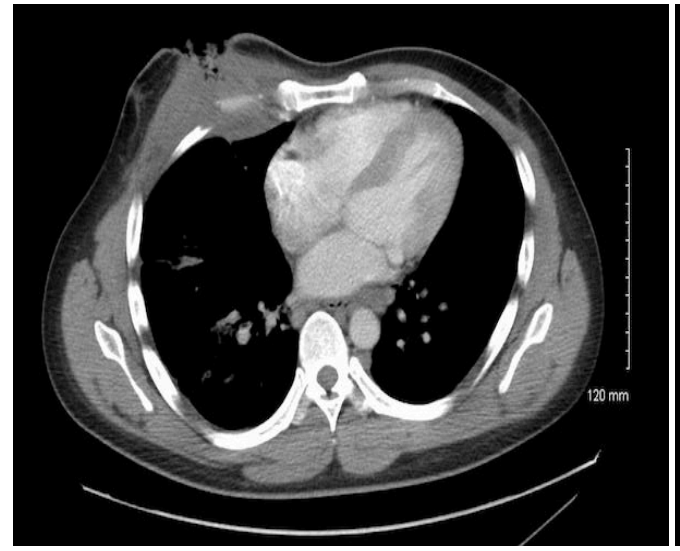

(a)

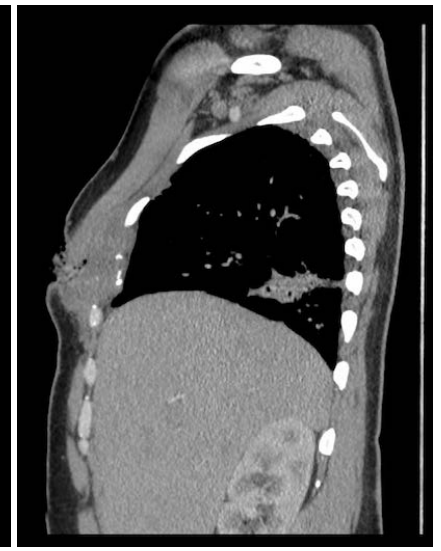

(b)

Figure 1. Cross-Sectional (a) and Saggital (b) CT with contrast-Images. Fracture site at the $4^{\text {th }}$ right rib (Red Arrow) surrounded by the abscess. Right Lung involvement can be appreciated due to abscess capsule extension. 
fractured his $4^{\text {th }}$ rib through a bout of cough that took place two to three weeks before the first visit. By the lack of medical intervention and the overlying infection, the fractured site became infected creating an abscess that was later aspirated and drained. Because the CMA, not the surgeon, was in closer contact with the patient while conducting dressing changes, it is possible that the disease transmission took place at that moment. As described before, wound management is a form of disease contraction [3], and it is likely that this was the mechanism attributed to such case, especially due to the lack of symptoms like coughing and sneezing.

$\mathrm{TB}$ can be symptomatically manifested in several ways, which might be misleading in occasions. A study conducted in Germany showed that $37.1 \%$ of their study population sought medical attention after noticing lymph node swelling and other $20 \%$ had osseous involvement as their primary clinical presentation. Only $14.3 \%$ of the patients presented with cough or respiratory issues [5].

This case presents a scenario in which the common symptomatic identifiers of TB (e.g. fever, chills, night sweats, cough) were not present, creating difficulty at the time of assigning a diagnosis. Moreover, the routine workup of laboratories, bacterial and fungal cultures were sent, yielding negative results, thereby adding further diagnostic uncertainty. The delayed CT scan worsened the clinical correlation of this presentation, hence limiting the use of proven preventative measures like placing surgical masks on patients to diminish transmission [6] [7].

Healthcare providers might be able to benefit from this case in several ways. Although it has been documented on multiple occasions, not all active TB patients have to present with the common symptoms of cough, fever or chills, which makes this case an example of an unexpected presentation. This report also intends to spread the different unusual mechanisms of TB contraction that are often times not considered, and to substantiate such mechanisms with this case presentation.

\section{Conclusion}

As it has been documented before, surgeons are known to avoid preventative measures whether it is by convenience or due to lack of time [4]. Also, given their schedules, it happens that often times they do not convey a full history and physical examination and just rely on the clinical picture they have on front. In unexpected cases like the one described, this might lead to transmission of infectious diseases thereby increasing the HW morbidity rates. Further effective communication and examination techniques should be devised to cope with the surgeon's growing schedules in order to eliminate suspicions of potential health hazards within the workplace.

\section{Acknowledgements}

Verbal informed consent was obtained. Patient granted permission to use his records as anonymous archival data. The contents of this article, opinions and 
conclusions are strictly those from the authors. All the authors have no conflict of interest to declare. Thank you to Robin Shealy for reviewing the literature and for the helpful comments during the manuscript preparation.

\section{Conflicts of Interest}

The authors declare no conflicts of interest regarding the publication of this paper.

\section{References}

[1] Centers for Disease Control and Prevention (CDC) (2015) Reported Tuberculosis in the United States. US Department of Health and Human Services, CDC, Atlanta, GA.

[2] Jones, R.M. (2017) Burden of Occupationally Acquired Pulmonary Tuberculosis among Healthcare Workers in the USA: A Risk Analysis. Annals of Work Exposures and Health, 61, 141-151. https://doi.org/10.1093/annweh/wxw015

[3] Nardell, E.A. (2016) Transmission and Institutional Infection Control of Tuberculosis. Cold Spring Harbor Perspectives in Medicine, 6, a018192. https://doi.org/10.1101/cshperspect.a018192

[4] Memon, A.G., Naeem, Z., Zaman, A. and Zahid, F. (2016) Occupational Health Related Concerns among Surgeons. International Journal of Health Sciences, 10, 279-291. https://doi.org/10.12816/0048819

[5] Singh, D.D., Vogel, M., Müller-Stöver, I., Scheich, T., Winzer, M., Göbels, S., Hüttig, F., Heinrich, S. MacKenzie, G., Jensen, B., Reuter, S., Häusinger, D. and Richter, J. (2011) TB or Not TB? Difficulties in the Diagnosis of Tuberculosis in HIV-Negative Immigrants to Germany. European Journal of Medical Research, 16, 381-384. https://doi.org/10.1186/2047-783X-16-9-381

[6] Dharmadhikari A.S., Mphahlele M., Stoltz A., Venter, K., Mathebula, R., Masotla, T., Lubbe, W., Pagano, M., First, M., Jensen, P.A., van der Walt, M. and Nardell, E.A. (2012) Surgical Face Masks Worn by Patients with Multidrug-Resistant Tuberculosis. American Journal of Respiratory and Critical Care Medicine, 185, 1104-1109. https://www.atsjournals.org/doi/abs/10.1164/rccm.201107-1190OC https://doi.org/10.1164/rccm.201107-1190OC

[7] Sim, S.W., Moey, K.S.P. and Tan, N.C. (2017) The Use of Facemasks to Prevent Respiratory Infection: A Literature Review in the Context of the Health Belief Model. Singapore Medical Journal, 55, 160-167. 